\title{
Gamma Knife radiosurgery for cluster-tic syndrome unresponsive to medical treatment: illustrative case
}

\author{
*Lina R. Barzaghi, MD, ${ }^{1}$ Edoardo Pompeo, MD, ${ }^{1}$ Luigi Albano, MD, ${ }^{1,2}$ Antonella Del Vecchio, $M{ }^{3},{ }^{3}$ and Pietro Mortini, $M D^{1}$ \\ Departments of ${ }^{1}$ Neurosurgery and Gamma Knife Radiosurgery; ${ }^{3}$ Medical Physics; and ${ }^{2}$ Neuroimaging Research Unit, Institute of Experimental Neurology, IRCCS San Raffaele \\ Scientific Institute, Vita-Salute University, Milan, Italy
}

\begin{abstract}
BACKGROUND Cluster-tic syndrome is a disorder characterized by the coexistence of symptoms related to both cluster headache and trigeminal neuralgia. Etiopathogenesis is not yet well defined. Medical treatment, including drugs for both cluster headache and trigeminal neuralgia, is the first therapeutic choice, whereas more invasive treatments are indicated in the case of pharmacological therapy failure or in the presence of drug side effects. To date, no randomized and/or large cohort trials describing Gamma Knife radiosurgery (GKRS) for cluster-tic syndrome are available, probably due to the syndrome's rarity.

OBSERVATIONS The authors describe the case of a 76-year-old woman with refractory cluster-tic syndrome who underwent GKRS with double target (the retrogasserian portion of the trigeminal nerve and the sphenopalatine ganglion). The Numerical Rating Scale (NRS) of pain and the Barrow Neurological Institute (BNI) pain intensity score before treatment were 7 (up to 10 during paroxysmal pain attacks) and V, respectively. At last follow-up, 24 months after GKRS, the patient had discontinued her pain medications and NRS and BNI pain scores were 1 and I, respectively. No trigeminal sensory disorders were reported.
\end{abstract}

LESSONS The present case shows that GKRS, in selected cases, could be an effective treatment in patients with refractory cluster-tic syndrome.

https://thejns.org/doi/abs/10.3171/CASE2191

KEYWORDS cluster-tic syndrome; cluster headache; facial pain; Gamma Knife radiosurgery; trigeminal neuralgia

Cluster-tic syndrome is a rare and disabling disorder characterized by simultaneous and ipsilateral occurrence of cluster headache attacks and trigeminal neuralgia, even though the two components may occur asynchronously. Although cluster-tic syndrome has been described since 1978, ${ }^{1}$ it was included in the International Classification of Headache Disorders only in its third edition (ICHD-3). ${ }^{2}$ In most cases described in medical literature, there was no obvious underlying structural brain lesion.

As stated in the ICHD-3, it is essential to recognize each condition distinctly to assess a correct diagnosis, since the two types of syndromes need separate treatments. Often, carbamazepine is effective for trigeminal neuralgia, whereas methysergide, lithium, and verapamil may be effective for cluster headache. As in other primary headaches, it may often be necessary to test multiple combinations of drugs and to adjust their dosages over time. Sometimes invasive procedures have been adopted for the treatment of refractory cluster-tic syndrome, such as section, thermocoagulation, and decompression of the trigeminal nerve in neurovascular conflicts, and even sellar and parasellar tumor removal has been reported to be associated with reduction of cluster frequency or pain severity. ${ }^{3-9}$

Gamma Knife radiosurgery (GKRS) owns a well-established role in the treatment of trigeminal neuralgia unresponsive to pain relief medication. ${ }^{10,11}$ On the contrary, GKRS has shown moderate efficacy in long-term pain control for cluster headache, with high rates

\footnotetext{
ABBREVIATIONS BNI = Barrow Neurological Institute; $\mathrm{CT}=$ computed tomography; GKRS = Gamma Knife radiosurgery; ICHD-3 = International Classification of Headache Disorders Third Edition; MRI = magnetic resonance imaging; NRS = Numerical Rating Scale; SPG = sphenopalatine ganglion; V1 = first trigeminal branch; $\mathrm{V} 2$ = second trigeminal branch.

INCLUDE WHEN CITING Published July 26, 2021; DOI: 10.3171/CASE2191.

SUBMITTED February 15, 2021. ACCEPTED February 18, 2021

${ }^{*}$ E.P. and L.A. contributed equally to this work.

(C) 2021 The authors, CC BY-NC-ND 4.0 (http://creativecommons.org/licenses/by-nc-nd/4.0/).
} 
of trigeminal nerve dysfunction. ${ }^{12-14}$ To the best of our knowledge, only one case report on GKRS for cluster-tic syndrome has been published. ${ }^{15}$ The authors described a complete resolution of pain, but severe trigeminal nerve dysfunction appeared a few months after treatment, causing clinical worsening. Conversely, we describe a case of cluster-tic syndrome, refractory to multimodality treatment, with an excellent outcome after GKRS.

\section{Illustrative Case \\ History}

A 76-year-old woman presented with refractory cluster-tic syndrome. The patient also reported being diagnosed with a nephritis, of unknown nature in her childhood, and tuberculosis (40 years before).

At the onset of symptoms, in 2014, the patient reported bouts of severe short-lasting unilateral left facial pain, located in the second trigeminal region (V2), described as sharp and sudden and triggered by chewing and cold water. The episodes lasted 1-2 minutes. Furthermore, she sometimes experienced attacks of left severe orbital and temporal pain associated with lacrimation, nasal congestion, and rhinorrhea lasting 20-120 minutes, occurring about once or twice per day for several weeks. Seasonal recurrence was initially described. Three years after the onset, she developed a persistent facial pain, located on the left frontoorbital, zygomatic, and maxillary regions (first trigeminal branch [V1] and V2), while still reporting attacks of excruciating pain with frontoorbital and temporal distribution associated with autonomic features. The diagnosis of persistent trigeminal neuralgia and episodic cluster headache was therefore made.

A cerebral magnetic resonance imaging (MRI) was performed in 2014 and 2019. A possible neurovascular conflict of the left trigeminal nerve with the ipsilateral superior cerebellar artery, without nerve displacement, was documented. No other pathological findings were identified.

She underwent multiple treatment courses with carbamazepine, lamotrigine, triptans, valproic acid, corticosteroids, oxygen, lithium, and verapamil, achieving only temporary improvements. She subsequently underwent topical lidocaine infiltration and neuromodulation with pulsate radiofrequency of the left infraorbital nerve, with transient and partial pain relief. However, she was unable to reduce her pharmacological therapy.

\section{Examination, Radiosurgical Procedure, and Postoperative Course}

At the time of presentation at our institution, the diagnosis of cluster-tic syndrome was confirmed. Pain intensity was assessed using the Barrow Neurological Institute (BNI) pain intensity score and the Numerical Rating Scale (NRS) of pain. The patient reported a BNI pain intensity value of $\mathrm{V}$, and her chronic headache was rated as 7 on the NRS, whereas paroxysms of pain were evaluated as 10 on the same scale, with daily attacks of autonomic features and no remission lasting for more than 1 year. No alterations at the general and neurological examination were identified.

The patient was taking a combination of pregabalin (150 mg 3 times a day), phenytoin (100 mg 2 times a day), and oxycodone (10 $\mathrm{mg} 2$ times a day), as well as tramadol as needed. We proposed GKRS or, as an alternative, microvascular decompression due to the patient's age and we explained the risks, potential adverse effects, and limitations of both treatments. She preferred GKRS due to its lower invasiveness.
After positioning a stereotactic Leksell $G$ frame (Leksell Elekta) under local anesthesia, a brain MRI scan (three-dimensional T1and T2-weighted sequences) and a thin-slice computed tomography (CT) scan of the head with localizer were obtained, coregistered, and fused using the GammaPlan software (Elekta Instruments AB). GKRS treatment was performed in January 2019 using the Leksell Gamma Knife Perfexion (Elekta Instrument AB).

We chose to target both the retrogasserian portion of the trigeminal nerve and the sphenopalatine ganglion (SPG). The latter was identified according to the previously described method using the Vidian canal on CT scan as an anatomical landmark. ${ }^{16}$ The maximum dose (isodose $100 \%$ ) for the trigeminal nerve and SPG was $80 \mathrm{~Gy}$ and $90 \mathrm{~Gy}$ via a 4-mm and 8-mm collimator, respectively. In reference to the trigeminal nerve target, the shot was placed 7.2 $\mathrm{mm}$ from the brainstem emergence of the trigeminal nerve, and the angle between the nerve and the pons was $43.6^{\circ}$. The maximal dose to $10 \mathrm{~mm}^{3}$ of the brainstem was $13.7 \mathrm{~Gy}$. The integral dose of the trigeminal nerve volume inside the $50 \%$ isodose line was 1.7 $\mathrm{mJ}$, whereas the dose rate was $1.822 \mathrm{~Gy} / \mathrm{min}$. The treatment plan is shown in Fig. 1.

Two weeks after treatment, the patient reported an initial improvement of pain, both chronic and paroxysmal, and no tramadol assumption was required. Oxycodone and phenytoin dosages were progressively reduced, and in April 2019, the patient was only taking pregabalin. Since then, the patient has reported complete pain relief and disappearance of autonomic features; therefore, in June 2019, pregabalin was discontinued.

At the last follow-up, on January 2021, 24 months after treatment, the patient reported being pain-free (BNI pain intensity score of I and NRS score of 1) despite complete discontinuation of pharmacological therapy. She underwent a brain MRI demonstrating no contrast enhancement on the trigeminal nerve or brain stem. Neither facial paresthesia nor sensory loss was reported.

\section{Discussion}

\section{Observations}

Due to its rarity, the pathophysiology underlying cluster-tic syndrome is still not well understood. Some authors have suggested that cooccurrence of trigeminal neuralgia and cluster headache does not happen by chance but implies a common pathophysiological substrate. ${ }^{17,18}$ On the other hand, Wilbrink et al. ${ }^{19}$ found that, among patients diagnosed with cluster headache, $4.5 \%$ reported attacks fulfilling the International Headache Society criteria for trigeminal neuralgia, a number exceeding trigeminal neuralgia's prevalence in the general population. The attacks often occurred in the V1 branch, which is less frequently affected in patients suffering from trigeminal neuralgia and was rarely evoked by typical triggers (chewing, pressure, etc.). The authors observed a good rate of symptom improvement with medications typically used for cluster headache and therefore rejected the hypothesis of a common underlying mechanism causing trigeminal neuralgia and cluster headache in their population. They interpreted the trigeminal pain observed in some of their patients as part of the cluster headache spectrum, suggesting a prominent role of the hypothalamus. ${ }^{20}$

In the absence of precise knowledge about the pathogenetic mechanism underlying cluster-tic syndrome, treatments for both trigeminal neuralgia and cluster headache are recommended. ${ }^{2}$ Among surgical treatments for cluster headache, the posterior hypothalamus and ventral tegmental area are targets for deep brain 

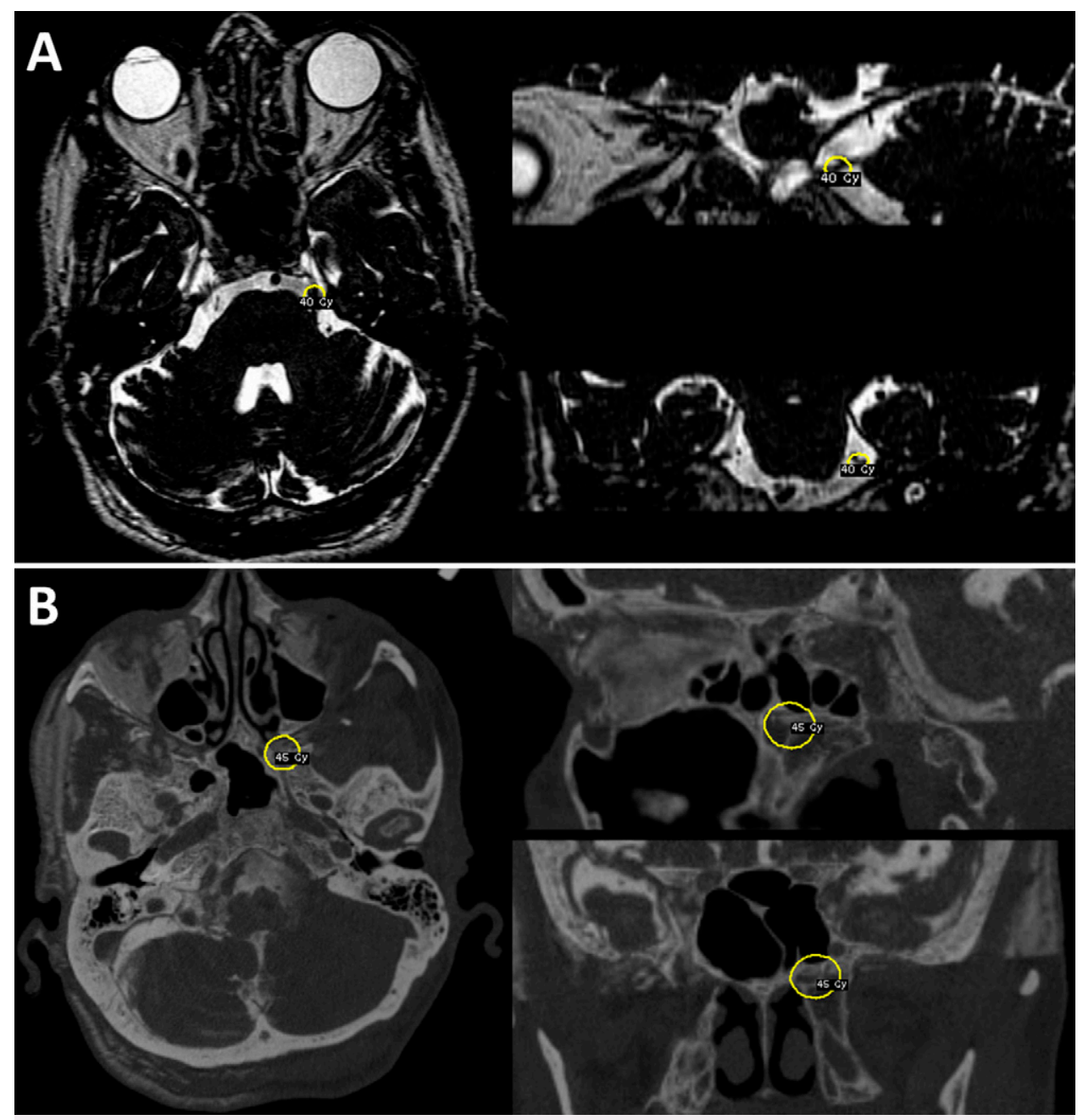

FIG. 1. GKRS treatment plan. A: MRI brain scans on axial, sagittal, and coronal planes of the left trigeminal target; the yellow circles shows the $50 \%$ isodose ( $40 \mathrm{~Gy}$, 4-mm collimator). B: MRI-CT fused brain images on axial, sagittal, and coronal planes of the left SPG target; the yellow circles shows the $50 \%$ isodose (45 Gy, 8-mm collimator).

stimulation, ${ }^{21}$ whereas, given the prominence of autonomic symptoms, invasive strategies have been employed for ablation or stimulation of the SPG, a major relay for both sympathetic and parasympathetic fibers. ${ }^{22}$ For this reason, both the trigeminal nerve and the SPG have been used simultaneously as targets in GKRS for cluster-tic syndrome.

To our knowledge, only one case of cluster-tic syndrome treated with GKRS has been reported in medical literature. ${ }^{15}$ Both the right trigeminal nerve and the SPG were targeted (with a maximum dose of 85 Gy and 90 Gy, respectively), similarly to our reported case (80 Gy and $90 \mathrm{~Gy})$. The female patient in the previous study experienced complete pain relief immediately after treatment. Six months later, she developed severe paresthesia and sensory loss in the right V1 and V2 regions. The right-sided pain was absent at the last follow-up (34 months), even if painful hypoesthesia was reported as a significant discomfort. For this reason, the authors concluded that radiosurgery for cluster-tic syndrome should not be recommended. ${ }^{15}$

Our experience differs from the case described above. Our patient not only reported complete resolution of pain and autonomic features despite discontinuation of medication but also appears not to be suffering from significant toxicity on the trigeminal nerve, dramatically improving her overall quality of life. Our experience is very interesting because it may overturn the interpretation from the literature that has been generally accepted until now.

GKRS has proven to be a safe and effective treatment for trigeminal neuralgia. In two of the largest published case series, Regis et al. ${ }^{10}$ and Kondziolka et al. ${ }^{11}$ reported a sensory dysfunction rate after radiosurgical treatment in $10.5 \%$ and $21.1 \%$ of patients, respectively, usually mild and not bothersome. Previous studies have shown variable and unclear GKRS effects on pain relief in cluster headache, reporting, on the other hand, high rates of posttreatment trigeminal dysfunction. ${ }^{12-14,23-25}$ Kano et al. ${ }^{14}$ reported their results after treatment of 17 patients with drug-refractory cluster headache, with a median follow-up of 34 months. Initial pain relief (BNI pain intensity scores of $|-| l \mid l b$ ) at a median of 2.9 months was observed in 12 patients. Among them, 2 patients had subsequent pain recurrence and 1 of them underwent repeat GKRS with acceptable results (BNI pain intensity score of I). Trigeminal 
dysfunction was reported in 8 patients $(47 \%)$, with 1 complaining of deafferentation pain. Sensory disfunction rate was the same in patients for whom the trigeminal nerve was targeted alone and in those for whom both the trigeminal nerve and SPG were targeted. Donnet et al. ${ }^{12}$ analyzed the outcome of GKRS in 10 patients with drug-resistant chronic cluster headache treated with a single retrogasserian target. After a median follow-up of 36.3 months, they observed symptom improvement in only 3 patients. Nine patients developed trigeminal sensory disfunctions, with 2 reporting deafferentation pain. The discrepancy in sensory disfunction between cluster headache and trigeminal neuralgia GKRS treatment has no established causative mechanism. A higher susceptibility of the trigeminal nerve to radiation in patients with cluster headache or, on the contrary, a lower susceptibility in patients with trigeminal neuralgia has been suggested. ${ }^{12}$ The previous reported case of cluster-tic syndrome treated by GKRS had led to the assumption that radiation susceptibility of the trigeminal nerve could be similar in cluster-tic syndrome and in cluster headache. Conversely, based on our experience, it is still not understood if increased trigeminal nerve susceptibility to radiation is present in cluster-tic syndrome.

To date, a significant role of the single patient's anatomy (e.g., trigeminal nerve length, pontotrigeminal angle) and treatment parameters (e.g., integral dose, dose rate, biologically effective dose) has been demonstrated in relation to the risk of developing trigeminal disfunction after radiosurgical treatment for trigeminal neuralgia. ${ }^{26,27}$ More specifically, Tuleasca et al. ${ }^{26}$ showed that when increasing the biologically effective dose, the risk of facial hypoesthesia increased exponentially; Barzaghi et al. ${ }^{27}$ reported a mean pontotrigeminal angle of $30^{\circ}$ in patients who experienced severe facial hypoesthesia after radiosurgery for trigeminal neuralgia, significantly smaller than in patients without trigeminal dysfunction development $\left(37^{\circ}\right)$. In our opinion, future studies focusing on these topics could be useful for better clarifying the effectiveness of GKRS and, even more, the risk for developing trigeminal nerve disfunction after treatment for both cluster-tic syndrome and cluster headache.

Furthermore, advanced brain MRI studies focusing on both the trigeminal nerve and the central nervous system are trying to determine structural and functional brain MRI modifications occurring in patients with trigeminal neuralgia and cluster headache in order to understand the etiology of these conditions and even a different susceptibility of the trigeminal nerve to radiation in patients with cluster-tic syndrome, cluster headache, and trigeminal neuralgia.

\section{Lessons}

The most important lesson of this study is that GKRS may be useful in patients suffering from refractory cluster-tic syndrome in selected cases. Our experience is encouraging; however, more data are needed to better understand how effective and how safe radiosurgery is in patients with cluster-tic. It is not possible to define, at the present time, if the trigeminal nerve in patients with cluster-tic is as susceptible to radiation as it appears to be in patients with cluster headache.

A possible limitation of our illustrative case is a shorter postoperative follow-up, albeit 24 months, compared with the report published by Donnet et al. ${ }^{15}$ Therefore, it could be that our positive results are due to an insufficient follow-up period, and clinical deterioration may be observed in the ensuing months. However, in the previously reported patient, trigeminal dysfunction already began to appear 6 months after treatment, and generally, in classical trigeminal neuralgia, it can develop within 24 months after GKRS. ${ }^{15}$

\section{References}

1. Green M, Apfelbaum R. Cluster-tic syndrome. Headache. 1978;18:112.

2. Headache Classification Committee of the International Headache Society (IHS). The International Classification of Headache Disorders, 3rd edition (beta version). Cephalalgia. 2013;33(9):629-808.

3. Watson P, Evans R. Cluster-tic syndrome. Headache. 1985;25(3): 123-126.

4. Alberca R, Ochoa JJ. Cluster tic syndrome. Neurology. 1994;44(6): 996-999.

5. Solomon S, Apfelbaum RI, Guglielmo KM. The cluster-tic syndrome and its surgical therapy. Cephalalgia. 1985;5(2):83-89.

6. Levyman C, Dagua Filho A dos S, Volpato MM, et al. Epidermoid tumour of the posterior fossa causing multiple facial pain-a case report. Cephalalgia. 1991;11(1):33-36.

7. Favier I, van Vliet JA, Roon KI, et al. Trigeminal autonomic cephaIgias due to structural lesions: a review of 31 cases. Arch Neurol. 2007;64(1):25-31.

8. de Coo I, van Dijk JMC, Metzemaekers JDM, Haan J. A case report about cluster-tic syndrome due to venous compression of the trigeminal nerve. Headache. 2017;57(4):654-657.

9. Leone M, Curone M, Mea E, Bussone G. Cluster-tic syndrome resolved by removal of pituitary adenoma: the first case. Cephalalgia. 2004;24(12):1088-1089.

10. Régis J, Tuleasca C, Resseguier N, et al. Long-term safety and efficacy of Gamma Knife surgery in classical trigeminal neuralgia: a 497-patient historical cohort study. J Neurosurg. 2016;124(4): 1079-1087.

11. Kondziolka D, Zorro O, Lobato-Polo J, et al. Gamma Knife stereotactic radiosurgery for idiopathic trigeminal neuralgia. $\mathrm{J}$ Neurosurg. 2010;112(4):758-765.

12. Donnet A, Tamura M, Valade D, Régis J. Trigeminal nerve radiosurgical treatment in intractable chronic cluster headache: unexpected high toxicity. Neurosurgery. 2006;59(6):1252-1257.

13. McClelland S III, Tendulkar RD, Barnett GH, et al. Long-term results of radiosurgery for refractory cluster headache. Neurosurgery. 2006;59(6):1258-1263.

14. Kano $\mathrm{H}$, Kondziolka D, Mathieu D, et al. Stereotactic radiosurgery for intractable cluster headache: an initial report from the North American Gamma Knife Consortium. J Neurosurg. 2011;114(6): 1736-1743.

15. Donnet A, Carron R, Régis J. Predilection to deafferentation pain syndrome after radiosurgery in cluster headache. Cephalalgia. 2012;32(8):635-640.

16. Alvernia JE, Spomar DG, Olivero WC. A computed tomography scan and anatomical cadaveric study of the pterygopalatine ganglion for use in Gamma Knife treatment of cluster headache. J Neurosurg. 2007;107(4):805-808.

17. Klimek A. Cluster-tic syndrome. Cephalalgia. 1987;7(2):161-162.

18. Monzillo PH, Sanvito WL, Da Costa AR. Cluster-tic syndrome: report of five new cases. Arq Neuropsiquiatr. 2000;58(2B):518-521.

19. Wilbrink LA, Weller CM, Cheung C, et al. Cluster-tic syndrome: a cross-sectional study of cluster headache patients. Headache. 2013;53(8):1334-1340.

20. May A, Bahra A, Büchel C, et al. Hypothalamic activation in cluster headache attacks. Lancet. 1998;352(9124):275-278.

21. Leone M, Franzini A, Broggi G, et al. Acute hypothalamic stimulation and ongoing cluster headache attacks. Neurology. 2006;67(10): 1844-1845.

22. Ho KWD, Przkora R, Kumar S. Sphenopalatine ganglion: block, radiofrequency ablation and neurostimulation - a systematic review. $J$ Headache Pain. 2017;18(1):118.

23. Ford RG, Ford KT, Swaid S, et al. Gamma knife treatment of refractory cluster headache. Headache. 1998;38(1):3-9. 
24. McClelland S III, Barnett GH, Neyman G, Suh JH. Repeat trigeminal nerve radiosurgery for refractory cluster headache fails to provide long-term pain relief. Headache. 2007;47(2): 298-300.

25. McDermott MW, ed. Radiosurgery: 8th International Stereotactic Radiosurgery Society Meeting, San Francisco, June 2007. Vol. 7. Karger; 2010:348-359.

26. Tuleasca C, Paddick I, Hopewell JW, et al. Establishment of a therapeutic ratio for Gamma Knife radiosurgery of trigeminal neuralgia: the critical importance of biologically effective dose versus physical dose. World Neurosurg. 2020;134(134):e204-e213.

27. Barzaghi LR, Albano L, Scudieri C, et al. Factors affecting long-lasting pain relief after Gamma Knife radiosurgery for trigeminal neuralgia: a single institutional analysis and literature review. Neurosurg Rev. Published online January 12, 2021. doi: 10.1007/s10143021-01474-9.

\section{Disclosures}

The authors report no conflict of interest concerning the materials or methods used in this study or the findings specified in this paper.

\section{Author Contributions}

Conception and design: Barzaghi, Albano. Acquisition of data: Barzaghi, Pompeo. Analysis and interpretation of data: Albano. Drafting the article: Barzaghi, Pompeo, Albano. Critically revising the article: Barzaghi, Pompeo, Albano, del Vecchio. Reviewed submitted version of manuscript: Barzaghi, del Vecchio. Approved the final version of the manuscript on behalf of all authors: Barzaghi. Administrative/technical/ material support: Barzaghi. Study supervision: Mortini.

\section{Correspondence}

Lina R. Barzaghi: IRCCS San Raffaele Scientific Institute, Milan, Italy. barzaghi.linaraffaella@hsr.it. 